\title{
Excitatory and inhibitory corticospinal responses to transcranial magnetic stimulation in patients with minor to moderate head injury
}

\author{
A V Chistyakov, J F Soustiel, H Hafner, M Trubnik, G Levy, M Feinsod
}

\begin{abstract}
Objectives-The changes in excitatory and inhibitory responses to transcranial magnetic stimulation (TMS), as attested by motor evoked potential (MEP) and silent period (SP) parameters, were compared in patients who sustained minor to moderate head injury.
\end{abstract}

Methods-A total of 38 patients with brain concussion, and diffuse, focal, and combined brain injury and 20 healthy volunteers were examined. The MEPs and SPs were recorded from the abductor pollicis brevis muscle after single pulse TMS 2 weeks after head trauma. The parameters assessed were the MEP resting threshold, the $M E P / M$ wave amplitude ratio, the central motor conduction time (CMCT), the SP threshold, the interthreshold difference (ITD), and the SP duration (SPD).

Results-The main finding was an increase in the ITD in patients with mild and moderate head injury due to the relatively greater augmentation of the MEP threshold. This was associated with a reduction of the MEP/M wave amplitude ratio. The degree of $M E P$ and SP changes depended on severity of head injury and was not related to the type of brain lesions. The SPD did not differ significantly in brain concussion, or diffuse, focal and combined brain injury groups compared with the control group. The CMCT was prolonged in patients with diffuse and combined brain lesions. Among subjective complaints only fatigue was significantly related to ITD, MEP, and SP threshold abnormalities.

Conclusions-The results suggest that mechanisms involved in MEP and SP generation are differently affected in head injury, the first being impaired more severely. The increase of the ITD accompanied by reduction of the MEP/M wave amplitude ratio may reflect a dissociated impairment of inhibitory and excitatory components of central motor control in head trauma.

(F Neurol Neurosurg Psychiatry 2001;70:580-587)

Keywords: head injury; silent period; motor evoked potential; transcranial magnetic stimulation

ondence to:

a chistyakov@

rambam.health.gov.il

Received 8 May 2000 and in revised form

1 November 2000

Accepted 15 November 2000
Transcranial magnetic stimulation (TMS) produces both excitatory and inhibitory effects on the human cerebral cortex. When applied over the motor cortex during voluntary target muscle contraction, TMS elicits a motor evoked potential (MEP) followed by a temporary suppression of the EMG activity, termed the cortical silent period (SP). Experimental findings suggest that at least the later part of the SP is due to excitation of intracortical inhibitory circuits or activation of descending inhibitory volley. ${ }^{1-3}$ The MEP amplitude, MEP threshold, and SP duration (SPD) have been widely used as parameters of cortical excitability in healthy subjects and patients in various clinical conditions. Recent reports have shown that excitatory and inhibitory responses to TMS can be differently affected by cortical ischaemic damage $^{4-6}$ and Parkinson's disease ${ }^{78}$ or independently modified by dopaminergic and antiepileptic drugs. ${ }^{9-13}$ Wassermann et $a l^{14}$ showed that the MEP and SP are produced by TMS on different threshold values and can be generated by different neural structures. In our previous study, ${ }^{15}$ we found an increased motor threshold to single pulse TMS and abnormal patterns of the MEP amplitude variability during slow rate repetitive TMS in patients with diffuse and focal brain lesions after head injury. We supposed that these MEP abnormalities reflected changes in the excitability of the motor cortex and may result from disturbed balance between excitatory and inhibitory intracortical activities. To our knowledge no data are available in the literature on the cortical silent period in head injury. Therefore, the aim of the present study was to further investigate excitability of the motor cortex in patients who sustained head trauma, analysing excitatory and inhibitory responses to TMS, and correlating these results with clinical and radiological findings.

Patients, materials, and methods SUBJECTS

Patients

Patients admitted to the hospital after traumatic head injury were selected for entry to the study on the basis of a combination of clinical, radiological, and electrophysiological criteria. Patients were included in the trial if their Glasgow coma score (GCS) on admission was between 9 and 15, their age was between 18 and 60 years, and they cooperated sufficiently for clinical and neurophysiological testing performed 2 weeks after head trauma. Patients were excluded on the grounds of (1) a concomitant cervical or a upper limb injury which could affect conduction along peripheral nerves and spinal cord; (2) an epidural or subdural haematoma or a skull fracture as they 
Table 1 Rate (\%) of most often found subjective complaints in head injury groups

\begin{tabular}{lllll}
\hline Subjective complaints & $\begin{array}{l}\text { Brain concussion } \\
\text { group }(n=9)\end{array}$ & $\begin{array}{l}\text { Diffuse brain injury } \\
\text { group }(n=7)\end{array}$ & $\begin{array}{l}\text { Focal lesion group } \\
(n=13)\end{array}$ & $\begin{array}{l}\text { Combined lesion } \\
\text { group }(n=9)\end{array}$ \\
\hline Fatigue & 22.2 & 28.6 & 46.2 & 55.6 \\
Memory and concentration disturbances & 44.4 & 57.1 & 61.5 & 55.6 \\
Dizziness & 33.3 & 42.9 & 38.5 & 55.6 \\
Headache & 44.4 & 14.3 & 46.2 & 33.3 \\
\hline
\end{tabular}

could decrease capacity of TMS to activate the cortex; (3) a history of spinal cord disease, cervical root compression, or peripheral neuropathy; and (4) electrophysiological evidence of subclinical radiculopathy or neuropathy (details in section Recording technique).

A total of 38 patients fulfilled the criteria for inclusion in the study. There were six women and 32 men with a mean age of 31.9 years (range 18-60). Ten patients sustained a minor head injury (GCS 15), 22 sustained a mild head injury (GCS 14 or 13), and six sustained a moderate head injury (GCS between 9 and 12). According to the type of brain injury, CT, and clinical findings, the patients were divided into four groups:

(1) Brain concussion group ( $n=9)$ : patients who sustained minor closed head injury with a brief transient unconsciousness followed by a complete recovery. Their GCS on admission was 15 and their CT did not disclose any structural cerebral injury.

(2) Diffuse brain injury group $(n=7)$ : patients who sustained mild $(\mathrm{n}=5)$ to moderate $(n=2)$ head injury (mean GCS on admission 12.6, range 9-14); CT findings in this group included subarachnoid haemorrhage and small punctiform parenchymal hemorrhages.

(3) Focal lesion group $(n=13)$ : patients who sustained minor $(n=1)$, mild $(n=11)$, and moderate $(n=1)$ head injury (mean GCS on admission 13.4 , range $10-14$ ) with radiological evidence of haemorrhagic brain contusion in the frontal or temporoparietal lobes. Parenchymal lesions were unilateral in nine patients and bilateral in four patients.

(4) Combined lesion group ( $n=9)$ : patients who sustained mild $(n=6)$ to moderate $(n=3)$ head injury (mean GCS on admission 12.7, range 10-14) with combined CT findings of diffuse axonal injury and brain contusions. The last were found unilaterally in three patients and bilaterally in six patients. There was no evidence of brain oedema or midline shift on initial CT.

A neurological examination performed by experienced neurologists 2 weeks after head trauma was normal in all patients with brain concussion or brain contusion. Two patients of the combined lesion group and one patient of the diffuse brain injury group had a slight to moderate hemiparesis.

For assessment of correlation between subjective complaints and electrophysiological findings, the patients were asked if they were presently experiencing symptoms related to the injury. Most frequent complaints were about disturbances in memory and concentration $(\mathrm{n}=21,55.3 \%)$, headache $(\mathrm{n}=14,36.8 \%)$, fatigue $(n=15,39.5 \%)$, and dizziness $(n=16$,
$42.1 \%)$. The distribution of subjective complaints among patients of different groups is shown in table 1 .

At the time of performing the electrophysiological tests all patients with moderate head injury and 19 of 22 patients with mild head injury were taking anticonvulsant medication (diphenylhydantoin (300 mg daily)). In one patient with minor head injury presenting with unilateral frontal contusion and in another three patients with mild head injury (two with bilateral focal lesions and one with subarachnoid haemorrhage) the anticonvulsant treatment was discontinued 6 days before electrophysiological examination. All patients of the brain concussion group were free from the influence of medication except for over the counter analgesics such as paracetamol.

\section{Controls}

Twenty age and sex matched healthy volunteers (mean age 33.2, range 20-54, 15 men, five women) participated in the study. They had no history or clinical evidence of neurological disease.

Informed consent for the study was obtained from all patients and control subjects. The protocol was approved by the local ethics committee.

\section{METHODS}

\section{Recording technique}

The subjects reclined on a comfortable bed in a quiet room. Motor evoked potentials (MEPs) and silent periods (SPs) were recorded using a Nicolet Viking 4 system (Nicolet Biomedical Ins, WI, USA) separately from each side. Filters were set between $2 \mathrm{~Hz}$ to $10 \mathrm{kHz}$ and impedance was kept below $5 \mathrm{k} \Omega$. Surface disc electrodes were placed over the abductor pollicis brevis (APB) muscle with a tendon belly montage. To maintain the correct level of voluntary isometric contraction or insure complete relaxation of the target muscle, EMG activity was continuously monitored, by subject and examiner, through audiovisual feedback.

\section{Transcranial magnetic stimulation}

Transcranial magnetic stimulation was performed with a conventional circular coil (outer diameter $9 \mathrm{~cm}$ ) connected with a Cadwell MES 10 magnetic stimulator (maximal intensity of 2 Tesla, pulse width of $70 \mu \mathrm{s}$, Cadwell Laboratories Inc, Kennewick, WA, USA). The coil was placed over the optimal scalp position for producing MEPs of maximal amplitude (lowest threshold) in the contralateral target muscle. This coil position was maintained fixed by manual handling throughout the TMS session. The initial current direction in the coil 
A

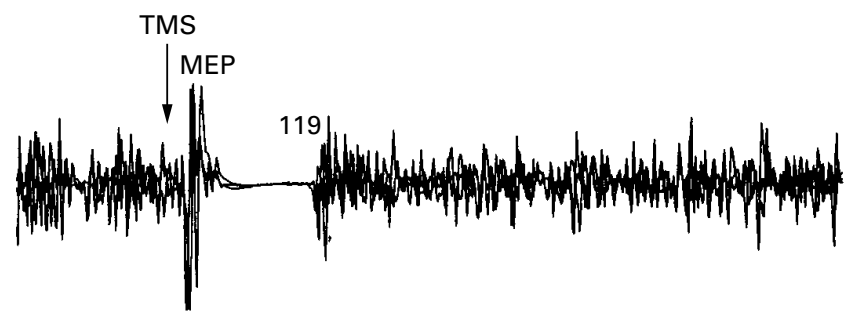

B
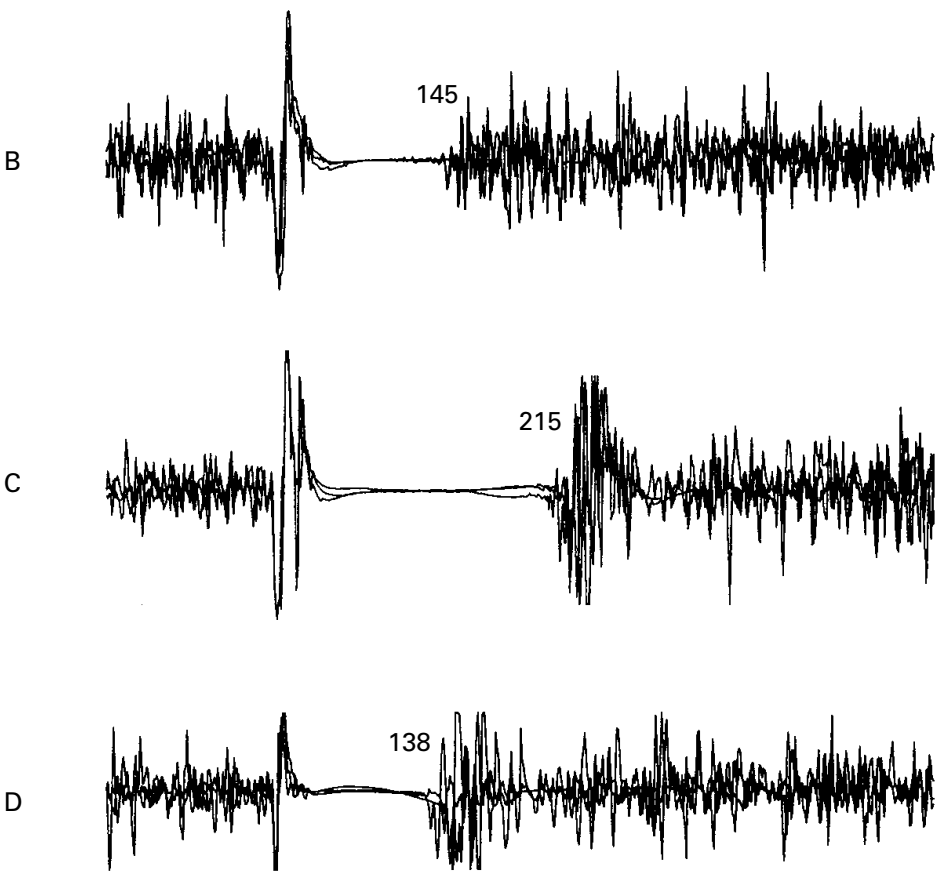

E
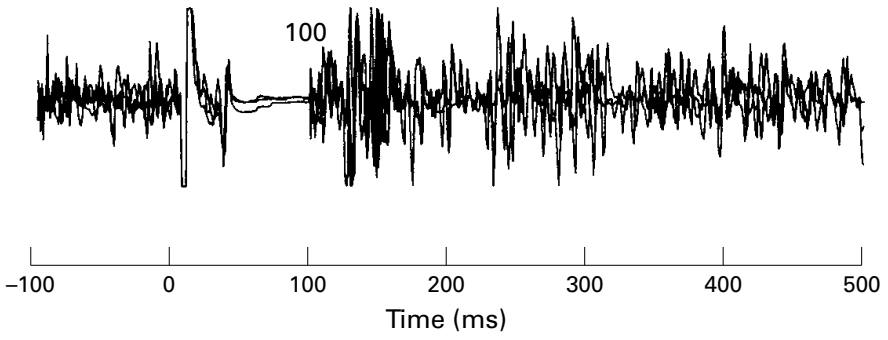

Figure 1 Typical set of silent periods recorded from the abductor pollicis brevis muscle in a normal subject at TMS intensities of $(A) 125 \%,(B) 150 \%$, and (C) $175 \%$ of the SP threshold, (D) $130 \%$ of the MEP resting threshold, and (E) to peripheral electrical stimulation of the median nerve. Three averaged SPs are superimposed for each trace. End of the SP (ms) is indicated by numbers.

was clockwise for stimulation of the right hemisphere and counterclockwise for the left hemisphere, ${ }^{16}$ although the biphasic current generated by the Cadwell stimulator results in a symmetric excitation of both hemispheres and MEPs are not influenced by the initial direction of the current in the coil. ${ }^{17}$

MEP and SP parameters

All MEP parameters-namely, the central motor conduction time (CMCT), the MEP/M wave amplitude ratio, the motor root conduction time (MRCT), and the MEP threshold were estimated during muscle relaxation. The CMCT was defined as the difference between the onset latency of the motor response to TMS and the peripheral latency (PL). The PL was determined by application of the formula: $\mathrm{PL}=1 / 2(\mathrm{M}+\mathrm{F}-1)$, where $\mathrm{M}$ is the latency of the $M$ (orthodromic) response ( $M$ wave) after supramaximal electrical stimulation of the median nerve at the wrist, and $\mathrm{F}$ is the shortest latency of $10 \mathrm{~F}$ waves (antidromic response). The MRCT was defined as the difference between the PL and the onset latency of the motor response to magnetic stimulation of the cervical motor roots at the level of $\mathrm{C} 7$ spinous process. ${ }^{18}$ Prolongation of the PL, the MRCT, or the onset latency to cervical magnetic stimulation beyond upper limits of the normal range was considered as evidence of peripheral conduction abnormalities. On the basis of the normal values previously defined in our laboratory, patients were excluded from the study if their PL for APB muscle was more than $17.1 \mathrm{~ms}$, the MRCT was above $2.2 \mathrm{~ms}$, and the onset MEP latency to magnetic stimulation of the cervical motor roots was more than $15.6 \mathrm{~ms}$.

The MEP amplitude was measured peak to peak at TMS intensity of $30 \%$ above the resting motor threshold (MEP threshold). The $\mathrm{MEP} / \mathrm{M}$ wave amplitude ratio was calculated by dividing the MEP amplitude by the maximal $M$ wave amplitude obtained after supramaximal peripheral electrical stimulation. The MEP/M wave amplitude ratio and the CMCT of each side were the result of averaging five consecutive trials.

The silent period was elicited while the subjects maintained a steady voluntary isometric contraction of the contralateral APB. Magnetic stimuli were delivered over the optimal motor cortex hand area (the same scalp position as for producing MEPs) at four levels of stimulus intensity: $125 \%, 150 \%$ and $175 \%$ of the SP threshold and $130 \%$ of the MEP threshold. The silent period duration (SPD) was measured from the MEP onset latency to the point when continuous EMG activity returned to its average level in the $100 \mathrm{~ms}$ before the stimulus. Three levels of muscular activation were evaluated: about $30 \%, 50 \%$, and $70 \%$ of maximal voluntary contraction. However, as no difference was found in individual SPDs recorded at different levels of contraction, results were aggregated. In total, nine trials were averaged for each stimulus intensity.

For assessment of spinal excitability, peripheral SP was recorded in response to supramaximal electrical stimulation of the median nerve. In addition, the mean $\mathrm{F} / \mathrm{M}$ wave amplitude ratio was calculated from 10 consecutive trials.

\section{Threshold determination}

The MEP threshold (motor resting threshold) was defined as the lowest stimulus intensity capable of eliciting in the relaxed APB at least five MEPs with an amplitude of at least $50 \mu \mathrm{V}$ in a series of 10 consecutive trials of TMS. The SP threshold (inhibition threshold) was defined as the lowest stimulus intensity that could produce the silent period lasting at least $50 \mathrm{~ms}$ in three consecutive trials while the subjects 
Table 2 SP and MEP parameters (mean (SD)) according to severity of head injuryt

\begin{tabular}{lllll}
\hline Parameters & $\begin{array}{l}\text { Control group } \\
(n=20)\end{array}$ & $\begin{array}{l}\text { Minor head } \\
\text { injury }(n=10)\end{array}$ & $\begin{array}{l}\text { Mild head injury } \\
(n=22)\end{array}$ & $\begin{array}{l}\text { Moderate head } \\
\text { injury }(n=6)\end{array}$ \\
\hline MEP threshold (motor resting threshold) & $40.2(6.1)$ & $42.3(4.3)$ & $55.9(11.6)^{\star}$ & $\begin{array}{l}60.7(11.9)^{\star} \\
\text { SP threshold (inhibition threshold) }\end{array}$ \\
Interthreshold difference & $34.2(5.2)$ & $36.1(3.8)$ & $43.2(6.5)^{\star}$ & $49.5(13.4)^{\star}$ \\
MEP/M wave amplitude ratio (\%) & $2.0(2.7)$ & $6.2(2.1)$ & $12.7(6.4)^{\star}$ & $11.2(4.0)$ \\
Central motor conduction time (ms) & $7.9(9.1)$ & $32.1(13.5)$ & $12.1(9.3)^{\star}$ & $15.3(12.7)^{\star}$ \\
Between side MEP threshold difference & $1.6(1.3)$ & $8.1(1.4)$ & $8.9(1.1)^{\star}$ & $8.7(2.0)$ \\
Between side SP threshold difference & $2.6(1.8)$ & $2.3(1.6)$ & $10.4(7.8)^{\star}$ & $5.5(3.8)$ \\
SP duration (ms) at intensity of 130\% MEP threshold & $150.8(37.0)$ & $1.5(1.3)$ & $3.2(2.9)$ & $3.0(2.8)$ \\
& & $151.9(25.7)$ & $196.1(35.8)^{\star}$ & $194.7(35.4)^{\star}$ \\
\hline
\end{tabular}

†Significant difference compared with control group, ${ }^{\star} \mathrm{p}<0.05$, ANOVA, $t$ test.

provided a voluntary contraction of about $50 \%$ of maximal EMG.

For assessment of the balance between excitatory and inhibitory central mechanisms, the interthreshold difference (ITD) was calculated as the difference between the MEP threshold and SP threshold.

DATA ANALYSIS AND STATISTICAL METHODS Although both sides were examined in every patient and healthy subject only data obtained from the "MEP worse" side were taken for statistical analysis. The criterion for selection was the highest MEP threshold or lowest MEP amplitude to cortical stimulation. The test results of the "MEP best" side were used for calculating the between side differences of MEP and SP parameters in the control and patient groups. In addition, the percentage of coincidence between the clinically affected side and "MEP worse" side was assessed in the group of 12 patients with unilateral cortical contusions (nine patients of the focal lesion group and three of the combined lesion group).

In multiple comparisons, the between group differences were estimated by the ANOVA and Bonferroni tests. In addition, Student's $t$ test was used when comparing the control and patient groups. Each parameter was considered abnormal whenever it exceeded the normal mean value by more than $2.5 \mathrm{SD}$. Between group comparisons of the frequencies of different nominally scaled variables were carried out by $\chi^{2}$ test. Pearson's correlation coefficients were calculated for relations between all independent measures.

\section{Results}

CONTROLS

Figure 1 shows a typical set of SPs recorded in a healthy subject at different intensities of TMS and to electrical stimulation of the median nerve. In the control group no between side asymmetry was found in excitatory and inhibitory responses. The SP threshold was lower than the MEP threshold in all subjects.

PATIENTS

MEPs and SPs in head injury of different severity Both MEP and SP thresholds were significantly increased in patients who sustained mild and moderate head injury (table 2). However, the increase of the SP threshold was much less pronounced than that of the MEP threshold. This resulted in a significant increase of the ITD, which was found abnormal in 12 patients $(54.5 \%)$ with mild brain injury and in three patients $(50.0 \%)$ with moderate brain injury.

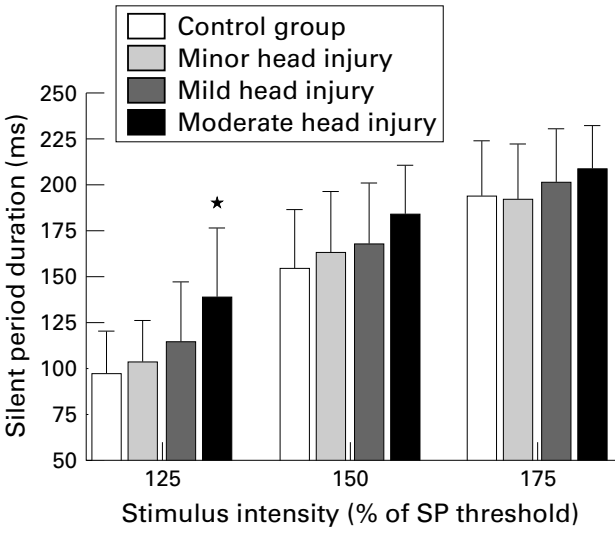

Figure 2 Silent period duration at stimulus intensities determined on the basis of the SP threshold in head injury of different severity in comparison with the control group $\left({ }^{\star} p<0.05\right)$.

Such a dissociated impairment of the MEP and SP was accompanied by a marked reduction in the $\mathrm{MEP} / \mathrm{M}$ wave amplitude ratio $(r=-0.4$, $\mathrm{p}<0.05$, table 2). Furthermore, by contrast with the SP threshold, the MEP threshold and the MEP/M wave amplitude ratio were abnormal predominantly on one side. Hence, these parameters showed an increased left-right asymmetry in 20 of the 28 patients with mild and moderate head injury $(71.4 \%)$. Selective unilateral increase of the MEP threshold was found in three patients of the mild head injury group in whom diphenylhydantoin treatment was discontinued 6 days before the examination and in another untreated patient of the minor head injury group. The CMCT tended to be slightly prolonged in all patient groups but reached significance compared with the control group only in the mild head injury group (table 2).

When measured at TMS intensity of $130 \%$ of the MEP threshold, the silent period duration (SPD) was found to be significantly prolonged in patients with mild and moderate head injury (table 2). However, at TMS intensities determined on the basis of the SP threshold only the SPD in patients with moderate head injury disclosed a significant difference for intensity of $125 \%$ of the SP threshold (fig 2).

The results of MEP and SP testing obtained in the minor head injury group did not differ from those in the control group (analysis of variance (ANOVA), Bonferroni test, and $t$ test, $\mathrm{p}>0.05)$. In this group, all MEP and SP parameters were within the normal range in all but one patient, whose between side MEP threshold difference was significantly increased. 
Table 3 SP and MEP parameters (mean (SD)) according to type of brain lesionst

\begin{tabular}{|c|c|c|c|c|c|}
\hline Parameters & Control group $(n=20)$ & $\begin{array}{l}\text { Brain concussion } \\
\text { group }(n=9)\end{array}$ & $\begin{array}{l}\text { Focal brain lesion } \\
\text { group }(n=13)\end{array}$ & $\begin{array}{l}\text { Diffuse brain injury } \\
\text { group }(n=7)\end{array}$ & $\begin{array}{l}\text { Combined lesion } \\
\text { group }(n=9)\end{array}$ \\
\hline MEP threshold (motor resting threshold) & $40.2(6.1)$ & $41.9(4.3)$ & $54.3(12.1)^{\star}$ & $56.7(11.1)^{\star}$ & $59.5(11.9)^{\star}$ \\
\hline SP threshold (inhibition threshold) & $34.2(5.2)$ & $35.6(3.7)$ & $42.0(6.7)^{\star}$ & $46.6(11.0)^{\star}$ & $46.0(8.7)^{\star}$ \\
\hline Interthreshold difference & $6.0(2.7)$ & $6.2(2.2)$ & $12.3(7.2)^{\star}$ & $10.1(5.2)^{\star}$ & $13.5(4.6)^{\star}$ \\
\hline $\mathrm{MEP} / \mathrm{M}$ wave amplitude ratio $(\%)$ & $29.9(9.1)$ & $33.1(13.9)$ & $11.9(8.6)^{\star}$ & $10.1(7.6)^{\star}$ & $17.2(12.7)^{\star}$ \\
\hline Central motor conduction time (ms) & $7.4(0.8)$ & $8.2(1.4)$ & $8.5(1.3)$ & $9.2(1.0)^{\star}$ & $9.1(1.7)^{\star}$ \\
\hline Between side MEP threshold difference & $1.6(1.3)$ & $2.4(1.6)$ & $9.1(6.8)^{\star}$ & $5.9(2.5)^{\star}$ & $11.5(10.1)^{\star}$ \\
\hline Between side SP threshold difference & $2.6(1.8)$ & $1.7(1.2)$ & $2.5(1.7)$ & $1.6(1.7)$ & $5.0(3.9)^{\star}$ \\
\hline SP duration (ms) at intensity of & $150.8(37.0)$ & $157.8(18.7)$ & $188.2(41.2)^{\star}$ & $190.3(50.4)$ & $199.0(30.3)^{\star}$ \\
\hline
\end{tabular}

†Significant difference compared with control group, ${ }^{\star} \mathrm{p}<0.05$, ANOVA, $t$ test.

$M E P$ and SPs related to radiological findings and subjective complaints

When MEP and SP findings were considered in relation to the type of brain lesion, significant changes compared with the control group were found for the MEP threshold, the SP threshold, the ITD, the $M E P / M$ wave amplitude ratio, and the between side MEP threshold difference in head injury groups which comprised patients with radiological evidence of focal or diffuse brain lesions (table 3). Multiple comparisons between these patient groups as well as comparisons between the brain concussion group and the control group did not show any significant differences (ANOVA and Bonferroni tests, $p>0.05$ ). Significant increase in the between side MEP threshold difference was found in nine patients $(69.2 \%)$ with focal brain injury, six patients $(85.7 \%)$ with diffuse brain injury, and six patients $(66.7 \%)$ with combined brain injury. By contrast, only one patient of the brain concussion group (11.1\%) showed an abnormal left-right asymmetry of the MEP threshold. Coincidence between the clinically affected side and "MEP worse" side was found in 11 of 12 patients with unilateral cortical contusions $(91.7 \%)$. One patient in whom the MEP threshold was slightly higher in the side opposite that of the focal cortical contusion presented also with signs of diffuse axonal injury on CT.

The CMCT was significantly prolonged in the diffuse and combined brain injury groups (table 3). Such a prolongation was associated with an increase in the MEP threshold $(r=0.49$, $\mathrm{p}<0.01)$. Five out of six patients whose CMCT exceeded the upper limit of the normal range had a significantly higher MEP threshold. Three of them also showed a marked increase in the SP threshold. No significant correlation was found between the CMCT and the SPD, or between the CMCT and the ITD.

The SPD as assessed at TMS intensities referred to the SP threshold did not differ significantly between the control and patient groups (fig 3). By contrast, the silent period recorded at TMS intensity of $130 \%$ of the MEP threshold was significantly prolonged in the focal and combined lesion groups compared with the control group (ANOVA, Bonferroni test, and t test, $\mathrm{p}<0.01$ ) and was significantly related to the ITD $(r=0.6$, $\mathrm{p}<0.001$ ).

In three patients presenting with hemiparesis the MEP threshold, the ITD, and the CMCT were abnormal on the affected side. Two of these patients had a significantly higher SP

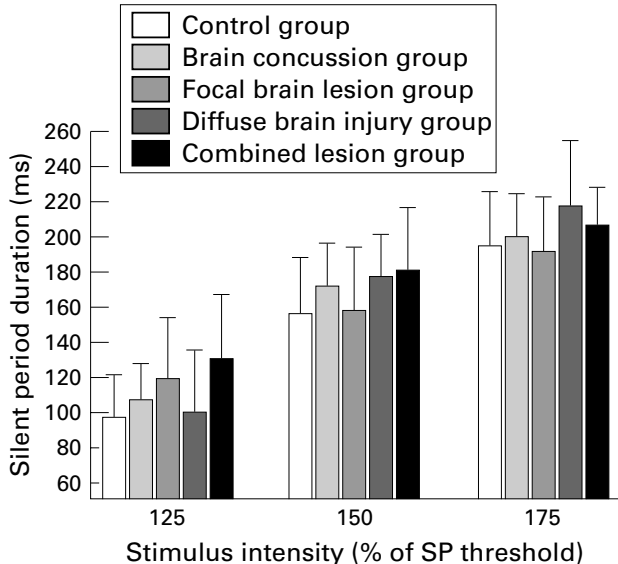

Figure 3 Silent period duration at stimulus intensities determined on the basis of the SP threshold in the control and head injury groups with different morphological patterns of brain lesions.

threshold, but normal SPDs at TMS intensities determined as a percentage of the SP threshold. Among subjective complaints only the rate of fatigue was significantly higher in patients who had an abnormal MEP threshold $(\mathrm{p}<0.05)$, SP threshold $(\mathrm{p}<0.001)$, and ITD ( $\mathrm{p}<0.01, \chi^{2}$ test).

For peripheral conduction and spinal excitability, the PL, the MRCT, the F/M wave amplitude ratio, and the peripheral silent period were normal in all patients.

\section{Discussion}

The main finding of this study was an increase in the ITD - that is, the difference between motor and inhibitory thresholds to TMS - in patients with mild and moderate head injury. This was associated with a significant reduction in the $\mathrm{MEP} / \mathrm{M}$ wave amplitude ratio. Before concluding that these changes reflect impaired excitability of the motor cortex due to head trauma, alternative explanations should be considered. A first explanation is related to anticonvulsant therapy, which was maintained during the study in mild and moderately head injured groups. Recent pharmacological data in healthy subjects showed that sodium and calcium channels blockers raise the MEP threshold and do not have effects on intracortical excitability and the SP. ${ }^{10}{ }^{13} 19$ As our patients who sustained focal, diffuse, and combined brain injury were receiving diphenylhydantoin, we cannot exclude the possibility that the selective increase in the MEP threshold was the result of diphenylhydantoin treatment. However, this alternative explanation seems unlikely for several reasons. Firstly, MEP abnormalities 
were observed unilaterally in most patients $(20$ of $29,68.9 \%$ ) whereas no between side asymmetry was found for SP parameters. Secondly, similar MEP changes were also found in four untreated patients with minor and mild head injury. Therefore, the dissociated change of MEPs and SPs cannot be exclusively attributed to the effect of anticonvulsant therapy although its influence cannot be totally ignored. A second possible explanation for our findings is altered spinal excitability due to cervical injury, which is often associated with head trauma. However, the normal peripheral SP and peripheral conduction (MRCT and PL), as well as the normal $\mathrm{F} / \mathrm{M}$ wave amplitude ratio found in all our patients excludes an impairment at spinal cord level and leaves two possible sites of change: cortical and subcortical.

The mechanism for increase of the MEP threshold in head injury is probably multifactorial and depends on the type and severity of the lesion. In this respect, the loss of the corticospinal neurons responding to TMS by repetitive firing and leading to generation of an excitatory postsynaptic potential (EPSP) in the spinal motor neurons may account for a higher MEP threshold in cortical contusions. On the other hand, slowing and desynchronisation of multiple descending volleys resulting in less effective temporal summation of EPSPs, which is necessary to bring the spinal motor neurons to firing threshold seems to be a major cause of the MEP threshold increase in axonal injuries. The reduction of the MEP/M wave amplitude ratio and increase of the CMCT found in patients with diffuse and combined brain lesions as well as a significant correlation between the CMCT and the MEP threshold support this assumption. The fact that the CMCT remained within the normal range in most patients $(n=32,84.2 \%)$ may be related to high intensities of TMS employed in patients with increased MEP threshold. Such stimulus intensities are probably capable of activating the corticospinal axons directly, thus "omitting" impairment at the cortical level. Together with trans-synaptic excitation of the residual pyramidal neurons, this may result in building up both I waves and D waves efficient for eliciting reproducible MEPs of normal latency. Another study of single motor unit discharges might help in examining this hypothesis.

By contrast with the MEP, the SP was much less affected in patients with head injury. Among SP parameters significant differences from the control group were found in the mild and moderate head injury groups for the SP threshold and the SPD measured at TMS intensity of $130 \%$ of the MEP threshold. The SPD recorded at TMS intensity of $125 \%$ of the SP threshold was slightly but significantly increased only in the moderate head injury group. It is commonly accepted that the later part of the SP (from $50 \mathrm{~ms}$ onwards) is certainly of some supraspinal origin. ${ }^{1-3}$ Therefore, it seems possible that the threshold for eliciting this part of the SP characterises the level of the excitability of supraspinal inhibitory systems. Whether it reflects activation of a descending inhibitory volley or interruption of the cortical drive by an inhibitory postsynaptic potential (IPSP) which is produced in the pyramidal cells by the GABAergic inhibitory neurons, or both, is still to be understood. To the best of our knowledge, there are no reports on the alteration of the SP threshold in patients and healthy subjects. In the patients of our series, the increase of the SP threshold was always associated with that of the MEP threshold but the changes of the second were more prominent. The underlying mechanism of this dissociation is unclear. It may now be speculated that the processes dominating in the MEP and the SP generation can be differently affected in head trauma. Although the cortical distribution maps of the SP and the MEP are not identical, ${ }^{14}{ }^{20}$ the areas of the human cortex from which inhibition and facilitation of the MEP could be obtained lie very close (within $1-2 \mathrm{~cm}$ ) to the corticospinal neurons. ${ }^{21}{ }^{22}$ It is unlikely that the neuronal elements responsible for evoking isolated MEPs and SPs would be selectively involved in the pathophysiology of the diffuse as well as the focal brain injury. Besides, changes in the neuronal membrane excitability of both excitatory and inhibitory sets of the neurons might induce similar fluctuations of the SP and MEP thresholds. However, as mentioned above, the MEP threshold, unlike the SP threshold, depends also on synchronised repetitive firing of the pyramidal tract neurons and the effective temporal summation of the EPSPs. This component of the MEP generation seems to be especially sensitive to the cortical or axonal injuries and, therefore, its impairment can lead to significant changes of the MEP independently from the SP.

For the silent period duration, we favour the idea that it is not a useful parameter for assessment of the excitability of the motor cortex in minor to moderate head injury. Numerous studies on the SP in motor disorders suggest that the SPD depends on the activity of subcortical structures, mainly basal ganglia, and the integrity of the connections between them and the motor cortex. The most common finding was a shortening of the SPD in Parkinson's disease which could be reversed after dopaminergic therapy and pallidotomy. ${ }^{823}$ Conversely, lengthening of the SPD without significant change in the MEP threshold has been found in Huntington's disease. ${ }^{24}$ Abnormal SPDs in patients with cortical and subcortical ischaemic damage have also been reported. Catano et $a l^{4}$ found a dissociated impairment of the SPD and the MEP amplitude in acute stroke. In another study, Classen et $a l^{6}$ found unilaterally prolonged SPDs in 16 of 174 patients presenting with hemiparetic subcortical stroke that spared the precentral gyrus and the pyramidal tract. The SPD lengthening was not accompanied by an increase of the MEP resting threshold or the SP threshold. The authors concluded that the lesions which affect the thalamocortical, striatocortical, and corticocortical nerve fibres and leave intact the primary motor cortex and the pyramidal tract could lead to disinhibition and 
overactivity of the GABAergic inhibitory neurons causing SPD prolongation. In the present study we found a significant increase of the SPD only in head injury of moderate severity and only at low TMS intensities $(125 \%$ of the SP threshold). Normal SPDs found in patients with minor and mild head injury as well as in patients with moderate head injury at intensities of $150 \%$ and $175 \%$ of the SP threshold point out the functional integrity of deeply located basal ganglia and suggest predominantly the cortical level of the lesions in our patients. There probably should be a more severe brain injury affecting both cortical and subcortical regions and their connections towards the motor cortex to cause significant changes in the SPD.

The rate of MEP and SP abnormalities in the mild and moderate head injury groups was significantly higher than in the minor head injury group. Similarly, SP and MEP changes were much more prominent in patients with morphological lesions seen on CT compared with patients with brain concussion. However, no significant difference was found in comparisons between different morphological patterns of brain lesions (focal, diffuse, and combined). In patients with pure unilateral contusions, the "MEP worse" side always coincided with the side of the lesion. However, in a patient with combined brain lesions the unilateral increase of the MEP threshold was found in the side opposite that of the focal contusion. Another manifestation of the discrepancy between radiological and electrophysiological findings was a high percentage $(85.7 \%)$ of increased between side MEP threshold difference in patients with diffuse brain injury. Given significant prolongation of the CMCT in the diffuse and combined lesion groups, it is most likely that unilateral MEP changes in these patient categories reflect concealed subclinical impairment of the central motor control on both neuronal (cortical excitability) and axonal (central motor conduction) levels. On the other hand, normal CMCTs found in patients of the focal brain injury group suggest that this type of brain lesion affects mainly intracortical mechanisms responsible for MEP generation. Further study in a larger number of patients and on the basis of the paired short interval TMS technique will be required to find out the role of impaired intracortical excitability in different brain lesions after head trauma.

In addition to the impairment of the central motor conduction, the disturbed balance between mechanisms of facilitation and inhibition of the cortical drive may account for some symptoms and subjective complaints reported 2 weeks after the accident. In patients with slight to moderate hemiparesis, the MEP threshold, the ITD, and the CMCT were significantly increased on the affected side. In patients whose neurological examination was normal, the rate of markedly increased ITDs, MEP, and SP thresholds was significantly higher among those patients who complained about enhanced exhaustion or fatigue. According to a recent study by Naalt et $a l^{25}$ fatigue is one of the most frequent subjective complaints reported by patients with mild and moderate head injury a month after the injury. This is in agreement with data obtained in our series in which symptoms of post-traumatic fatigue were found in 15 of the 38 patients (39.5\%). Ten of these patients had an abnormal ITD and MEP threshold and nine had an abnormal SP threshold $(\mathrm{p}<0.01)$. No correlation was found between electrophysiological abnormalities and disturbances in memory and concentration, headache, and dizziness. These results suggest that significant changes of the ITD, MEP, and SP thresholds may be used as supportive evidence of impairment of central motor function in patients with post-traumatic fatigue syndrome.

Our finding of the dissociated change of the MEP and SP thresholds may be of particular importance from a methodological point of view. In previous clinical studies, it has been customary to adjust the stimulus intensity in recording of both the MEP and the SP with respect to the individual MEP resting threshold value. However, the use of the MEP threshold as a baseline parameter for evaluation of inhibitory responses may be inadequate in those situations when it is increased independently of the SP threshold - that is, when TMS produces dissociated excitatory and inhibitory effects, the first being deficient and the second being basically intact or even enhanced. The disproportional change of the MEP and SP thresholds may lead to eliciting excessively prolonged SPDs at stimulus intensities determined as a percentage of MEP resting threshold values. In fact, in the present study the SP recorded at TMS intensity of $130 \%$ of the MEP threshold was found to be significantly prolonged in both mild and moderate head injury groups and its duration (SPD) was strongly correlated with the ITD in all patient groups $(\mathrm{p}<0.001)$. However, when measured at TMS intensities of $125 \%, 150 \%$, and $175 \%$ of the SP threshold, the SPD did not show any significant difference between the control and mild head injury groups and was not related to the ITD. Therefore, the lengthening of the SPD at intensities that were determined on the basis of the MEP threshold most likely reflects a decreased excitability of the motor cortex rather than its exaggerated inhibition. The discrepancy in the results of the paired pulse and silent period tests reported by Liepert et $a l^{26}$ confirms this conclusion. The authors demonstrated a reduced intracortical inhibition in the affected hemisphere in patients after acute stroke using a paired pulse paradigm. On the other hand, an enhanced cortical inhibition as evidenced by the unilateral prolongation of the SPD was found in the same patients. In that study, the silent period was recorded at an intensity of $150 \%$ of the motor resting threshold. As the second was considerably increased in the affected hemisphere, the intensity of the single pulse TMS applied to this side was significantly higher than that of the unaffected side. Such a between side intensity difference could be a possible reason for the unilateral lengthening of the silent period despite the cortical disinhibition shown by the paired pulse 
test. Our data suggest that the SP threshold is a more appropriate reference for measuring the SPD provided that possible changes in spinal excitability, which may affect the initial part of the SP, are taken into consideration. We think that the assessment of the SP threshold may be also relevant in studies in which repetitive TMS is employed for treatment of psychiatric disorders and epilepsy. ${ }^{27}{ }^{28}$ As the antidepressant and anticonvulsive therapy may exert dissociated influences on the excitatory and inhibitory responses, the between threshold difference should be taken into consideration before choosing the individual intensity of repetitive TMS as well as for monitoring the changes in cortical excitability during the treatment.

In conclusion, the results of the present study show that diffuse, focal, and combined brain injuries of mild and moderate severity affect the SP to a lesser extent than the MEP. The increase in the ITD accompanied by reduction of the $\mathrm{MEP} / \mathrm{M}$ wave amplitude ratio suggests dissociated impairment of inhibitory and excitatory components of the central motor control in head trauma.

This study was supported by the generosity of the Joseph Szydlowsky Foundation. We are greatly indebted to the commitment of Mrs S Stern.

1 Fuhr P, Agostino R, Hallett M. Spinal motor neuron excitability during the silent period after cortical stimulation. ability during the silent period after cortical stimulation
Electroencephalogr Clin Neurophysiol 1991;81:257-62.

2 Cantello R, Gianelli M, Civardi C, et al. Magnetic brain stimulation: the silent period after the motor evoked potenstimulation: the silent period after
tial. Neurology 1992;42:1951-9.

3 Roick H, Giesen HJ, Benecke R. On the origin of the postexcitatory inhibition seen after transcranial magnetic brain stimulation in awake human subjects. Exp Brain Res 1993;94:489-98.

4 Catano A, Houa $M$, Noel P. Magnetic transcranial stimulation: dissociation of excitatory and inhibitory mechanisms in acute strokes. Electroencephalogr Clin Neurophysiol 1997;105:29-36.

5 Catano A, Houa M, Noel P. Magnetic transcranial stimulation: clinical interest of silent period in acute and chronic stages of stroke. Electroencephalogr Clin Neurophysiol 1997;105:290-6.

6 Classen J, Schnitzler A, Binkofski F, et al. The motor syndrome associated with exaggerated inhibition within the primary motor cortex of patients with hemiparetic stroke. Brain 1997;120:605-19.

7 Valzania F, Strafella AP, Quatrale R, et al. Motor evoked responses to paired cortical magnetic stimulation in responses to paired cortical magnetic stimulation in 1996;105:37-43.

8 Young MS, Triggs WJ, Bowers D, et al. Stereotactic pallidotomy lengthens the transcranial magnetic cortical stimulation silent period in Parkinson's disease. Neurology 1997;49:1278-83.
9 Priori A, Berardelli A, Inghilleri M, et al. Motor cortical inhibition and the dopaminergic system. Brain 1994;117: 317-23.

10 Mavroudakis N, Caroyer JM, Brunko E, et al. Effects of diphenylhydantoin on motor potentials evoked with magnetic stimulation. Electroencephalogr Clin Neurophysiol 1994;93:428-33.

11 Inghilleri M, Berardelli A, Marchetti P, et al. Effects of diazepam, baclofen and thiopental on the silent period evoked by transcranial magnetic stimulation in humans. Exp Brain Res 1996;109:467-72.

12 Schulze-Bonhage A, Knott H, Ferbert A. Effects of cabamazepine on cortical excitability and inhibitory phenomena: a study with paired transcranial magnetic stimulation. Electroencephalogr Clin Neurophysiol 1996;99: 267-73.

13 Chen R, Samii A, Canos M, et al. Effects of phenytoin on cortical excitability in humans. Neurology 1997;49:881-3.

14 Wassermann EM, Pascual-Leone A, Valls-Sole J, et al. Topography of the inhibitory and excitatory responses to transcranial magnetic stimulation in a hand muscle. Electroencephalogr Clin Neurophysiol 1993;89:424-33.

15 Chistyakov AV, Hafner H, Soustiel JF, et al. Dissociation of somatosensory and motor evoked potentials in noncomatose patients after head injury. Clin Neurophysiol 1999;110:1080-9.

16 Chiappa KH, Cros D, Cohen D. Magnetic stimulation: determination of coil current flow direction. Neurology 1991;42:1154-5.

17 Claus D, Murray NMF, Spitzer A, et al. The influence of stimulus type on the magnetic excitation of nerve structures. Electroencephalogr Clin Neurophysiol 1991;81: 342-9.

18 Chistyakov AV, Soustiel JF, Hafner H, et al. Motor and somatosensory conduction in cervical myelopathy and radiculopathy. Spine 1995;20:2135-40.

19 Ziemann U, Lonnecker S, Steinhoff BJ, et al. Effects of antiepileptic drugs on motor cortex excitability in humans: a transcranial magnetic stimulation study. Ann Neurol 1996; 40:367-8.

20 Lewko JP, Stokic DS, Tarkka IM. Dissociation of cortical areas responsible for evoking excitatory and inhibitory responses in the small hand muscles. Brain Topogr 1996,8: 397-405.

21 Davey NJ, Romaiguere P, Maskill DW, et al. Suppression of voluntary motor activity revealed using transcranial magnetic stimulation of the motor cortex in man. $f$ Physiol 1994;477:223-35.

22 Ashby P, Reynolds C, Wennberg R, et al. On the focal nature of inhibition and facilitation in the human motor cortex. Clin Neurophysiol 1999;110:550-5.

23 Valls-Sole J, Pascual-Leone A, Brazil-Neto JP, et al. Abnormal facilitation of the response to transcranial magnetic stimulation in patients with Parkinson's disease. Neurology 1994;44:735-41.

24 Priori A, Berardelli A, Inghilleri M, et al. Electromyographic silent period after transcranial brain stimulation in Huntington's disease. Mov Disord 1994;9:178-82.

25 Naalt van der J, Zomeren van AH, Sluiter WJ, et al. One year outcome in mild to moderate head injury: the predictive value of acute injury characteristics related to complaints and return to work. F Neurol Neurosurg Psychiatry 1999;66: 207-13.

26 Liepert J, Storch P, Fritsch A, et al. Motor cortex disinhibition in acute stroke. Clin Neurophysiol 2000;111;671-6.

27 Chen R, Classen J, Georloff C, et al. Depression of motor cortex excitability by low frequency transcranial magnetic cortex excitability by low frequency transcr
stimulation. Neurology $1997 \mathrm{~b} ; 48: 1398-403$.

28 George MS, Lisanby SH, Sackeim HA. Transcranial magnetic stimulation: applications in neuropsychiatry. Arch Gen Psychiatry 1999;6:300-11. 